\title{
Updated Guidelines for the Diagnosis of Human Brucellosis - China, 2019
}

\author{
Hai Jiang'; Lan Feng'; Jinxing Lu $^{1, *}$
}

Brucellosis is a neglected infection that has a widespread geographic distribution. Based on an evaluation from the World Health Organization (WHO), brucellosis cases have been reported in more than 170 countries with about 500,000 new cases being reported each year. However, the actual number of brucellosis patients is much higher, and it is believed to be approximately 10-25 times the number of reported cases (1). This big discrepancy between the reported rate and the actual incidence rate is largely due to misdiagnosis and underdiagnosis, especially in endemic areas. With the aim of improving the diagnostic capacity, the National Health Commission of China released its updated Guidelines for the Diagnosis of Human Brucellosis (WS 269-2019) on July 1, 2019. Compared with the old guidelines (WS 269-2007), there are three major changes in the new guidelines including new detection methods, revised classification of brucellosis stages, and biosafety requirements. In particular, the new guidelines mention lateral flow assays (LFA) as one of the new detection methods that are expected to provide fast and simple point-of-care testing at county-level clinics and CDC labs.

\section{BACKGROUND}

The classification of brucellosis by the WHO, US CDC, and China CDC differ as shown in Table 1. According to WS 269-2019 (the updated guidelines of China CDC), brucellosis cases are classified as suspected, probable (clinically diagnosed), confirmed (laboratory confirmed), and covert infection. Probable cases of brucellosis are diagnosed based on a combination of epidemiologic exposure, clinical manifestations, and positive results of presumptive laboratory tests including the Rose Bengal test (RBT), enzyme-linked immunosorbent assay (ELISA), LFA, and Gram staining of Brucella spp. Confirmed cases are probable cases with a positive result for one of the following tests: standard agglutination test (SAT), complement fixation test (CFT), Coombs test, or isolation of Brucella spp. In contrast, the WHO only classifies cases as suspected, probable, and confirmed, and does not list LFA, Gram staining, or CFT as diagnostic tests. Similarly, US CDC only classifies cases as probable and confirmed, and while it does not include RBT, CFT, Gram staining, or LFA, it does include PCR and BMAT.

The definitive diagnosis of brucellosis requires isolation of the pathogen from blood or other tissue and body fluids; however, the inability to isolate the pathogen does not rule out the infection. In contrast to bacterial culture, serological testing is more sensitive and, therefore, preferred in routine clinical practice. The main serology methods currently used in China are serum agglutination tests. One such test is RBT, which is a practical screening test that has low diagnostic specificity but is used in combination with SAT, a commonly-used test that is used as a confirmatory test. However, there is a lack of consensus about the diagnostic endpoint titer: the WHO and US CDC have set the minimum antibody endpoint titer at 1:160, but the minimum antibody endpoint titer in China is $1: 100$. When the result of the SAT test is suspicious, the Coombs test is recommended for confirmation as it can improve the accuracy of diagnosis. However, because the Coombs test kit has not been licensed by the China Food and Drug Administration (CFDA), it is not used in clinical or medical institutions and is only used for research.

ELISA (IgG and/or IgM) is another serological test that is sensitive, fast, and convenient for the detection of brucellosis. It shows high sensitivity and specificity and should be used as a routine lab test when brucellosis is suspected in clinical practice. LFA, another serological test, does not require extensive laboratory infrastructure or technical expertise, and compared to the standard SAT and/or culture, the sensitivity and specificity are higher at $92 \%-95 \%$ and $97 \%$, respectively, in endemic settings (2). Thus, it seems that there are several viable options with regard to serological testing for the diagnosis of brucellosis. Therefore, based on newly published studies and 
TABLE 1. Definition and classification of brucellosis by China CDC, WHO, and US CDC.

\begin{tabular}{|c|c|c|c|}
\hline Case classification & China CDC & WHO & US CDC \\
\hline Suspected case & $\begin{array}{l}\text { Epidemiologic exposure+clinical } \\
\text { manifestations }\end{array}$ & $\begin{array}{l}\text { Epidemiologic exposure+clinical } \\
\text { manifestations }\end{array}$ & None \\
\hline Probable case & $\begin{array}{l}\text { Suspected case+presumptive } \\
\text { diagnosis (RBT, ELISA, LFA, } \\
\text { Gram staining of Brucella spp.) }\end{array}$ & $\begin{array}{l}\text { Suspected case+presumptive } \\
\text { diagnosis }(R B T+S A T \geq 160)\end{array}$ & $\begin{array}{l}\text { Epidemiologic exposure+clinical } \\
\text { manifestations+presumptive diagnosis (SAT } \geq \\
160, \text { BMAT, PCR) }\end{array}$ \\
\hline Confirmed case & $\begin{array}{l}\text { Probable case+confirmatory } \\
\text { diagnosis (SAT } \geq 100 \text {, Coombs } \\
\text { IgG, CFT, Culture) }\end{array}$ & $\begin{array}{l}\text { Probable case+confirmatory } \\
\text { diagnosis (ELISA IgG, Coombs } \\
\text { lgG, Culture) }\end{array}$ & $\begin{array}{l}\text { Probable case+confirmatory diagnosis (a } \\
\text { fourfold or higher increase in Brucella antibody } \\
\text { titer between acute- and convalescent-phase } \\
\text { serum specimens obtained } \geq 2 \text { weeks apart; } \\
\text { Culture) }\end{array}$ \\
\hline Covert infection & $\begin{array}{l}\text { Epidemiologic } \\
\text { exposure+confirmatory } \\
\text { diagnosis+asymptomatic } \\
\text { manifestations }\end{array}$ & None & $x_{0}$ \\
\hline
\end{tabular}

clinical practices, China CDC initiated a process to assess the performance of new tests with the support of the National Health Commission.

\section{METHODS OF ASSESSMENT}

China CDC considered several factors and the opinions of many experts in determining whether to update its guidelines. First, China CDC referred to a systematic review that was conducted before the creation of the WS 269-2019 guidelines: the review covered 157 studies and 716,280 samples from 25 provincial-level administrative divisions (PLADs) and covered the period 1954-2012 (3). China CDC also invited 8 provincial experts to act as reviewers of the collected evidence and the updated WS269-2019 recommendations. The reviewers were selected based on their expertise in Brucella infection, diagnosis, or the intersection of these topics. The reviewers participated in several conferences with China CDC staff members from 2015 to 2019, and during this period, China CDC staff members presented the process and outcome of the updated WS 269-2019 recommendations. The participants provided their individual input about (1) whether the updated evidence might influence clinical practice in China, and (2) how the updated WS 269-2019 recommendations might translate to clinical practice in China. After the final conference, China CDC assessed the evidence, the updated WS 269-2019 recommendations, and the individual perspectives provided by the expert reviewers.

\section{RATIONALE AND EVIDENCE}

The findings of the systematic review showed that RBT and SAT were the most widely used in county- levels of CDC labs. LFA and ELISA are not only in keeping with international criteria but also have high concordance with SAT. With regard to the development of future tests, CDC has conducted a comprehensive evaluation of three approved LFA kits. The internal report generated based on the evaluation indicated that there was no significant difference between the results of the kits and SAT according to the results of the kappa and $\chi^{2}$ tests $(p>0.05)$. Another study showed that among 235 patients with brucellosis, $232(98.7 \%)$ tested positive with ELISA (4). Based on this finding, it was concluded that ELISA has higher sensitivity and specificity for the detection of brucellosis than the agglutination test, as reported in other previous studies (5-7). This means that its performance is "substantially equivalent to or better than" that of the currently commercially available predictive tests.

\section{RECOMMENDATIONS}

LFA and ELISA were cleared by the CFDA and were considered acceptable alternatives for the laboratory diagnosis of brucellosis. Based on the criteria established by the 2019 Diagnostic Guidelines for Human Brucellosis, clinicians and laboratories should consider serological tests to be cleared by the CFDA as China CDC-recommended procedures for the serodiagnosis of brucellosis. Considering the importance of the disease, the international brucellosis research community should focus on viable diagnostic options. Therefore, I will highlight below a small selection of the recently reported advanced tests. Fluorescence polarization assay seems to be a valuable method for the diagnosis of brucellosis in humans (8). Another option is MALDI-TOF mass spectrometry, which is revolutionizing the clinical diagnosis 
procedure (9). Additionally, China CDC has developed a spectral database for the identification of Brucella called the Pathogen Identification System. The system is now used in developed provincial-level CDCs and is becoming the diagnostic choice in modern clinical laboratories in China. It is expected that the advances in diagnostic technology will lead to the standardization of brucellosis guidelines in the future.

Conflicts of interest: The authors declare that they have no conflicts of interest.

Funding: This study was funded by Major Infectious Diseases such as AIDS and Viral Hepatitis Prevention and Control technology major projects grant 2018ZX10712-001.

doi: $10.46234 / \mathrm{ccdcw} 2020.129$

\# Corresponding author: Jinxing Lu, lujinxing@icdc.cn.

${ }^{1}$ State Key Laboratory for Infectious Disease Prevention and Control, Collaborative Innovation Center for Diagnosis and Treatment of Infectious Diseases, National Institute for Communicable Disease Control and Prevention, Chinese Center for Disease Control and Prevention, Beijing, China.

Submitted: April 27, 2020; Accepted: May 27, 2020

\section{REFERENCES}

1. Pappas G, Papadimitriou P, Akritidis N, Christou L, Tsianos EV. The new global map of human brucellosis. Lancet Infect Dis 2006;6(2):91 -
9. http://dx.doi.org/10.1016/S1473-3099(06)70382-6.

2. Mizanbayeva S, Smits HL, Zhalilova K, Abdoel TH, Kozakov S, Ospanov KS, et al. The evaluation of a user-friendly lateral flow assay for the serodiagnosis of human brucellosis in Kazakhstan. Diagn Microbiol Infect Dis 2009;65(1):14 - 20. http://dx.doi.org/10.1016/j.diagmicrobio. 2009.05.002.

3. Liu X, Jiang H, Tian GZ, Li XW, Wang DL, Zhang QX, et al. Systematic literature review of diagnostic test methods for human brucellosis used in China from 2004 to 2014. Chin J Endemiol 2015;34(12):920 - 5. http://dx.doi.org/10.3760/cma.j.issn.2095-4255. 2015.12.018. (In Chinese).

4. Xu NN, Wang W, Chen FZ, Li W, Wang G. ELISA is superior to bacterial culture and agglutination test in the diagnosis of brucellosis in an endemic area in China. BMC Infect Dis 2020;20(1):11. http://dx.doi.org/10.1186/s12879-019-4729-1.

5. Gad El-Rab MO, Kambal AM. Evaluation of a Brucelle enzyme immunoassay test (ELISA) in comparison with bacteriological culture and agglutination. J Infect 1998;36(2):197 - 201. http://dx.doi.org/ 10.1016/S0163-4453(98)80013-3.

6. Osoba AO, Balkhy H, Memish Z, Khan MY, Al-Thagafi A, Al Shareef B, et al. Diagnostic value of Brucella ELISA IgG and IgM in bacteremic and non-bacteremic patients with brucellosis. J Chemother 2001;13(S1):54 9. http://dx.doi.org/10.1080/1120009X.2001.11782330.

7. Ulu-Kilic A, Metan G, Alp E. Clinical presentations and diagnosis of brucellosis. Recent Pat Antiinfect Drug Discov 2013;8(1):34-41. http://dx.doi.org/10.2174/1574891X11308010007.

8. Konstantinidis A, Minas A, Pournaras S, Kansouzidou A, Papastergiou P, Maniatis A, et al. Evaluation and comparison of fluorescence polarization assay with three of the currently used serological tests in diagnosis of human brucellosis. Eur J Clin Microbiol Infect Dis 2007;26(10):715 21. http://dx.doi.org/10.1007/s10096-007-0363-8.

9. Mesureur J, Arend S, Cellière B, Courault P, Cotte-Pattat PJ, Totty H, et al. A MALDI-TOF MS database with broad genus coverage for specieslevel identification of Brucella. PLoS Negl Trop Dis 2018;12(10): e0006874. http://dx.doi.org/10.1371/journal.pntd.0006874. 